\title{
Almost Periodic Sequence Solutions of a Discrete Predator-Prey System with Beddington-DeAngelis Functional Response
}

\author{
Zengji Du and Wenbin Li \\ School of Mathematics and Statistics, Jiangsu Normal University, Xuzhou, Jiangsu 221116, China \\ Correspondence should be addressed to Zengji Du; duzengji@163.com
}

Received 21 April 2014; Accepted 12 June 2014; Published 17 July 2014

Academic Editor: Kunquan Lan

Copyright ( 2014 Z. Du and W. Li. This is an open access article distributed under the Creative Commons Attribution License, which permits unrestricted use, distribution, and reproduction in any medium, provided the original work is properly cited.

This paper considers a discrete predator-prey system with Beddington-DeAngelis functional response. Sufficient conditions are obtained for the existence of the almost periodic solution which is uniformly asymptotically stable by constructing a Lyapunov function.

\section{Introduction}

In the past decades, the predator-prey competition models have been extensively studied by many authors (see [1-7]). Many excellent works have been done for the predatorprey model with functional response, such as Zhu and Wang [8] who considered a Volterra model with modified Leslie-Gower Holling-type II schemes. Cai et al. [9] studied the positive periodic solution for a multispecies competition-predator system with the Holling III functional and time delays. A well-known model of such systems is the predator-prey model with a Beddington-DeAngelis functional response which was originally proposed by Beddington [10] and DeAngelis et al. [11], independently. The dynamics of this model is described by the following differential equations:

$$
\begin{gathered}
\dot{x}=r x(t)\left[1-\frac{x(t)}{K}\right]-\frac{b x(t) y(t)}{1+n x(t)+m y(t)}, \\
\dot{y}=y(t)\left[-d+\frac{f b x(t)}{1+n x(t)+m y(t)}\right],
\end{gathered}
$$

where $x$ and $y$ represent prey and predator densities, respectively. Usually, the constant $r$ is called intrinsic growth rate of the prey; $K$ is the carrying capacity of the prey; $b$ and $n$ are positive constants that describe the effects of capture rate and handling time, respectively, on the feeding rate; $d$ is the death rate of the predator; $f$ is the birth rate of the predator; $m \geq 0$ (units: 1/predator) is a constant describing the magnitude of interference among predators. Many excellent works have been done for the predator-prey system with a BeddingtonDeAngelis functional response, such as Fan and Kuang [12], Liu et al. [13], and Baek [14].

It has been found that discrete time models governed by difference equations are more appropriate than the continuous ones when the populations have nonoverlapping generations. Discrete time models can also provide efficient computational models of continuous models for numerical simulations. There are some existing results on discrete predator-prey systems [15-17]. For example, in [15], by using the method of upper and lower solutions and the degree theory the authors have studied the existence of periodic positive solutions for a competitive system with two parameters. Zhang and Wang [16] studied the following discrete model:

$$
\begin{array}{r}
x(k+1)=x(k) \exp [a(k)-b(k) x(k) \\
\left.\quad-\frac{c(k) y(k)}{\alpha(k)+\beta(k) x(k)+\gamma(k) y(k)}\right], \\
y(k+1) \quad y(k) \exp [-d(k) \\
\left.\quad+\frac{f(k) y(k)}{\alpha(k)+\beta(k) x(k)+\gamma(k) y(k)}\right] .
\end{array}
$$


The authors used the Mawhin's coincidence degree theory to obtain some sufficient conditions for the existence of positive solutions.

But, nowadays, models with almost periodic coefficient have drawn more attention. Li and Chen [18] considered the almost periodic solutions of the following discrete almost periodic logistic equation:

$$
x(n+1)=x(n) \exp \left\{r(n)\left(1-\frac{x(n)}{K(n)}\right)\right\} .
$$

Niu and Chen [19] proposed the following model:

$$
\begin{aligned}
& x_{1}(n+1) \\
& =x_{1}(n) \\
& \times \exp \left[r_{1}(n)\right. \\
& \left.\quad \times\left(1-\frac{x_{1}(n)}{K_{1}(n)}-\mu_{2}(n) x_{2}(n)-b_{1}(n) \mu_{1}(n)\right)\right], \\
& x_{2}(n+1) \quad \\
& =x_{2}(n) \quad\left[r_{2}(n)\right. \\
& \left.\times \exp \left(1-\frac{x_{2}(n)}{K_{2}(n)}-\mu_{1}(n) x_{1}(n)-b_{2}(n) \mu_{2}(n)\right)\right], \\
& \Delta \mu_{1}(n)=-\alpha_{1}(n) \mu_{1}(n)+\beta_{1}(n) x_{1}(n), \\
& \Delta \mu_{2}(n)=-\alpha_{2}(n) \mu_{2}(n)+\beta_{2}(n) x_{2}(n) .
\end{aligned}
$$

By constructing a suitable Lyapunov function, they obtained the existence and uniqueness of the almost periodic solution which is uniformly asymptotically stable.

Li et al. [20] studied the following system:

$$
\begin{aligned}
& x(k+1) \\
& =x(k) \\
& \times \exp \{a(k)-b(k) x(k) \\
& \left.\quad-p(k, x(k), y(k), x(k-\mu), y(k-v)) \frac{y(k)}{x(k)}\right\}, \\
& y(k+1)=y(k) \exp \left\{c(k)-\frac{d(k) y(k)}{x(k-\mu)}\right\} .
\end{aligned}
$$

With the method of the theory of difference inequality and constructing a suitable Lyapunov function, they obtained the permanence and the almost periodic solution of the system.
Inspired by the above papers, in this paper, we consider the following discrete predator-prey system:

$$
\begin{aligned}
& x_{1}(n+1) \\
& =x_{1}(n) \\
& \times \exp \left[r_{1}(n)\left(1-\frac{x_{1}(n)}{K_{1}(n)}\right)\right. \\
& \left.-\frac{b(n) x_{2}(n)}{\alpha(n)+\beta(n) x_{1}(n)+\gamma(n) x_{2}(n)}\right] \\
& x_{2}(n+1) \\
& =x_{2}(n) \\
& \times \exp \left[-r_{2}(n)\right. \\
& \left.+\frac{c(n) x_{1}(n)}{\alpha(n)+\beta(n) x_{1}(n)+\gamma(n) x_{2}(n)}\right] \text {, }
\end{aligned}
$$

where $x_{1}(n)$ and $x_{2}(n)$ represent prey and predator densities at time $t$.

From the point of view of biology, we assumed that the initial conditions of (6) are of the form

$$
x_{i}(0)>0, \quad i=1,2 \text {. }
$$

Then, it is easy to see that the solutions of (6) with the initial condition (7) are defined and remain positive for all $n \in Z^{+}$. Throughout this paper, for any bounded sequence $\{a(n)\}$, we denote $a^{M}=\sup _{n \in N}\{a(n)\}$ and $a^{L}=\inf _{n \in N}\{a(n)\}$, and we assume that

$\left(\mathrm{H}_{1}\right)\left\{r_{1}(n)\right\},\left\{r_{2}(n)\right\},\{K(n)\},\{b(n)\},\{c(n)\},\{\alpha(n)\}$, $\{\beta(n)\}$, and $\{\gamma(n)\}$ are bounded nonnegative almost periodic sequences such that

$$
\begin{array}{ll}
0<r_{1}^{L} \leq r_{1}(n) \leq r_{1}^{M}, & 0<r_{2}^{L} \leq r_{2}(n) \leq r_{2}^{M}, \\
0<K^{L} \leq K(n) \leq K^{M}, & 0<\alpha^{L} \leq \alpha(n) \leq \alpha^{M}, \\
0<\beta^{L} \leq \beta(n) \leq \beta^{M}, & 0<\gamma^{L} \leq \gamma(n) \leq \gamma^{M}, \\
0<b^{L} \leq b(n) \leq b^{M}, & 0<c^{L} \leq c(n) \leq c^{M}, \\
\left(\mathrm{H}_{2}\right) r_{1}^{L}-\left(b^{M} / \gamma^{L}\right)>0, & \\
\left(\mathrm{H}_{3}\right)\left(c^{L} / \beta^{M}\right)\left(1-\alpha^{M}\right)-r_{2}^{M}>0 .
\end{array}
$$

The organization of this paper is as follows. In Section 2, we will introduce some definitions and several useful definitions and lemmas. In Section 3, by applying the theory of difference inequality, we get the permanence of system (6). In Section 4, by constructing a suitable Lyapunov function, we obtain the existence of the almost periodic solution for system (6) which is uniformly asymptotically stable. Finally, we give some examples and numerical simulations to verify our results. 


\section{Preliminaries}

In this section, we will introduce some basic definitions and several useful lemmas.

Definition 1. System (6) is said to be permanent if there exist positive constants $M_{i}, m_{i}$, which are independent of the solutions of the system, such that any positive solution $\left(x_{1}(n), x_{2}(n)\right)^{\mathrm{T}}$ of system (6) satisfies

$$
m_{i} \leq \lim _{n \rightarrow+\infty} \inf x_{i}(n) \leq \lim _{n \rightarrow+\infty} \sup x_{i}(n) \leq M_{i}, \quad i=1,2 .
$$

Lemma 2 (see [21]). Assume that $\{x(n)\}$ satisfies $x(n)>0$ and

$$
x(n+1) \leq x(n) \exp \{a(n)-b(n) x(n)\}, \quad n \in N,
$$

where $a(n)$ and $b(n)$ are nonnegative sequences bounded above and below by positive constants. Then,

$$
\lim _{n \rightarrow+\infty} \sup x(n) \leq \frac{1}{b^{L}} \exp \left\{a^{M}-1\right\} .
$$

Lemma 3 (see [21]). Assume that $\{x(n)\}$ satisfies $x(n)>0$ and

$$
x(n+1) \geq x(n) \exp \{a(n)-b(n) x(n)\}, \quad n \geq N_{0},
$$

and $\lim _{n \rightarrow+\infty} \sup x(n) \leq x^{*}, x\left(N_{0}\right)>0$, where $a(n)$ and $b(n)$ are nonnegative sequences bounded above and below by positive constants and $N_{0} \in N$. Then,

$$
\lim _{n \rightarrow+\infty} \inf x(n) \geq \min \left\{\frac{a^{L}}{b^{M}} \exp \left\{a^{M}-b^{M} x^{*}\right\}, \frac{a^{L}}{b^{M}}\right\} .
$$

Lemma 4 (see [22]). Let $x(n)$ and $b(n)$ be nonnegative sequences defined on $N$, and $c \geq 0$ is a constant. If

$$
x(n) \leq c+\sum_{s=0}^{n-1} b(s) x(s), \quad n \in N,
$$

then

$$
x(n) \leq c \prod_{s=0}^{n-1}[1+b(s)], \quad n \in N
$$

Definition 5 (see [23]). A sequence $x: Z \rightarrow R$ is called an almost periodic sequence if the $\epsilon$-translation number set of $x$

$$
E\{\epsilon, x\}=\{\tau \in Z:|x(n+\tau)-x(n)|<\epsilon, \forall n \in Z\}
$$

is a relatively dense set in $Z$ for all $\epsilon>0$; that is, for any given $\epsilon>0$, there exists an integer $l(\epsilon)>0$ such that each interval of length $l(\epsilon)$ contains an integer $\tau \in E\{\epsilon, x\}$ such that

$$
|x(n+\tau)-x(n)|<\epsilon, \quad \forall n \in Z,
$$

$\tau$ is called the $\epsilon$-translation number or $\epsilon$-almost period.

Definition 6 (see [23]). Let $f: Z \times D \rightarrow R$, where $D$ is an open set in $R ; f(n, x)$ is said to be almost periodic in $n$ uniformly for $x \in D$, or uniformly almost periodic for short, if, for any $\epsilon>0$ and any compact set $S$ in $D$, there exists a positive integer $l(\epsilon, S)$ such that any interval of length $l(\epsilon, S)$ contains a integer $\tau$ for which

$$
|f(n+\tau, x)-f(n, x)|<\epsilon,
$$

for all $n \in Z$ and $x \in S$. $\tau$ is called the $\epsilon$-translation number of $f(n, x)$.

Lemma 7 (see [23]). $\{x(n)\}$ is an almost periodic sequence if and only iffor any sequence $\left\{m_{i}\right\} \subset Z$ there exists a subsequence $\left\{m_{i_{k}}\right\} \subset\left\{m_{i}\right\}$ such that $x\left(n+m_{i_{k}}\right)$ converges uniformly on $n \in Z$ as $k \rightarrow \infty$. Furthermore, the limit sequence is also an almost periodic sequence.

In [24], Zhang considered the following almost periodic difference system:

$$
x(n+1)=f(n, x(n)), \quad n \in Z^{+},
$$

where $f: Z \times S_{B} \rightarrow R, S_{B}=\{x \in R:\|x\|<B\}$, and $f(n, x)$ is almost periodic in $n$ uniformly for $x \in S_{B}$ and is continuous in $x$. The product system of (19) is the following system:

$$
x(n+1)=f(n, x(n)), \quad y(n+1)=f(n, y(n)),
$$

and the following lemma is obtained.

Lemma 8 (see [24]). Suppose that there exists a Lyapunov functional $V(n, x, y)$ defined for $n \in Z^{+},\|x\|<B,\|y\|<B$ satisfying the following conditions:

$\left[\mathrm{C}_{1}\right] a(\|x-y\|) \leq V(n, x, y) \leq b(\|x-y\|)$, where $a, b \in$ $\widehat{T}$ with

$$
\widehat{T}=\left\{a \in C\left(R^{+}, R^{+}\right): a(0)=0 \text { and } a \text { is increasing }\right\},
$$

$\left[\mathrm{C}_{2}\right]\left|V\left(n, x_{1}, y_{1}\right)-V\left(n, x_{2}, y_{2}\right)\right| \leq L\left(\left\|x_{1}-x_{2}\right\|+\| y_{1}-\right.$ $\left.y_{2} \|\right)$, where $L>0$ is a constant,

$\left[\mathrm{C}_{3}\right] \Delta V_{(20)} \leq-\lambda V(n, x, y)$, where $0<\lambda<1$ is a constant and

$$
\Delta V_{(20)}=V(n+1, f(n, x), f(n, y))-V(n, x, y) \text {. }
$$

Moreover, if there exists a solution $\varphi(n)$ of system (20) such that $\|\varphi(n)\|<B^{*}<B$ for $n \in Z^{+}$, then there exists a unique uniformly asymptotically stable almost periodic solution $p(n)$ of system (20) which is bounded by $B^{*}$. In particular, if $f(n, x)$ is $\omega$-periodic function, then there exists a unique uniformly asymptotically stable $\omega$-periodic solution of (20).

\section{Permanence}

Theorem 9. Assume that $\left(H_{1}\right),\left(H_{2}\right)$, and $\left(H_{3}\right)$ hold, then every solution $\left(x_{1}(n), x_{2}(n)\right)^{\mathrm{T}}$ of system (6) satisfies

$$
x_{i *} \leq \lim _{n \rightarrow+\infty} \inf x_{i}(n) \leq \lim _{n \rightarrow+\infty} \sup x_{i}(n) \leq x_{i}^{*}, \quad i=1,2 .
$$


That is, system (6) is permanent, where

$$
\begin{gathered}
x_{1}^{*}=\frac{K^{M}}{r_{1}^{L}} \exp \left\{r_{1}^{M}-1\right\}, \\
x_{1 *}=\frac{K^{L}\left(r_{1}^{L} \gamma^{L}-b^{M}\right)}{\gamma^{L} r_{1}^{M}} \exp \left\{r_{1}^{L}-\frac{b^{M}}{\gamma^{L}}-\frac{r_{1}^{M}}{K^{L}} x_{1}^{*}\right\}, \\
x_{2}^{*}=\exp \left\{2\left(\frac{c^{M}}{\beta^{L}}-r_{2}^{L}\right)\right\}, \\
x_{2 *}=\min \left\{T \exp \left\{\frac{c^{L}\left(1-\alpha^{M}\right)-r_{2}^{M} \beta^{M}}{\beta^{M}}-\frac{c^{M} \gamma^{M}}{\beta^{L}} x_{2}^{*}\right\}, T\right\} \\
T=\frac{\beta^{L} c^{L}\left(1-\alpha^{M}\right)-\beta^{L} \beta^{M} r_{2}^{M}}{c^{M} \gamma^{M} \beta^{M}} .
\end{gathered}
$$

Proof. Let $\left(x_{1}(n), x_{2}(n)\right)^{\mathrm{T}}$ be any positive solution of system (6); from the first equation of system (6), it follows that

$$
x_{1}(n+1) \leq x_{1}(n) \exp \left\{r_{1}(n)-\frac{r_{1}(n)}{K(n)} x_{1}(n)\right\} \text {. }
$$

Thus, as a direct corollary of Lemma 2, it follows that

$$
\lim _{n \rightarrow+\infty} \sup x_{1}(n) \leq \frac{K^{M}}{r_{1}^{L}} \exp \left\{r_{1}^{M}-1\right\}:=x_{1}^{*}
$$

On the other hand, from the first equation of system (6), we obtain

$$
\begin{aligned}
x_{1}(n+1) & \geq x_{1}(n) \exp \left\{r_{1}(n)-\frac{r_{1}(n)}{K(n)} x_{1}(n)-\frac{b(n) x_{2}(n)}{\gamma(n) x_{2}(n)}\right\} \\
& =x_{1}(n) \exp \left\{r_{1}(n)-\frac{b(n)}{\gamma(n)}-\frac{r_{1}(n)}{K(n)} x_{1}(n)\right\} .
\end{aligned}
$$

According to Lemma 3 and assumption $\left(\mathrm{H}_{2}\right)$, we have

$$
\begin{aligned}
& \lim _{n \rightarrow+\infty} \inf x_{1}(n) \\
& \geq \min \left\{\frac{K^{L}\left(r_{1}^{L} \gamma^{L}-b^{M}\right)}{\gamma^{L} r_{1}^{M}}\right. \\
&\left.\quad \times \exp \left\{r_{1}^{L}-\frac{b^{M}}{\gamma^{L}}-\frac{r_{1}^{M}}{K^{L}} x_{1}^{*}\right\}, \frac{K^{L}\left(r_{1}^{L} \gamma^{L}-b^{M}\right)}{\gamma^{L} r_{1}^{M}}\right\} .
\end{aligned}
$$

Notice that

$$
\begin{aligned}
\frac{r_{1}^{M} / K^{L}}{r_{1}^{L}-b^{M} / \gamma^{L}} \cdot x_{1}^{*} & =\frac{r_{1}^{M} / K^{L}}{r_{1}^{L}-b^{M} / \gamma^{L}} \cdot \frac{K^{M}}{r_{1}^{L}} \exp \left\{r_{1}^{M}-1\right\} \\
& =\frac{r_{1}^{M}}{r_{1}^{L}} \cdot \frac{K^{M}}{K^{L}} \cdot \frac{\exp \left\{r_{1}^{M}-1\right\}}{r_{1}^{L}-b^{M} / \gamma^{L}} \\
& >\frac{r_{1}^{M}}{r_{1}^{L}} \cdot \frac{K^{M}}{K^{L}} \cdot \frac{r_{1}^{M}}{r_{1}^{L}} \\
& >1 .
\end{aligned}
$$

In the above inequality, using the Bernoulli inequality $e^{x}>$ $1+x$, for $x>-1$, one has

$$
\begin{aligned}
& \lim _{n \rightarrow+\infty} \inf x_{1}(n) \\
& \quad \geq \frac{K^{L}\left(r_{1}^{L} \gamma^{L}-b^{M}\right)}{\gamma^{L} r_{1}^{M}} \exp \left\{r_{1}^{L}-\frac{b^{M}}{\gamma^{L}}-\frac{r_{1}^{M}}{K^{L}} x_{1}^{*}\right\}:=x_{1 *} .
\end{aligned}
$$

From the second equation of system (6), we can obtain

$$
x_{2}(n+1) \leq x_{2}(n) \exp \left\{-r_{2}(n)+\frac{c(n)}{\beta(n)}\right\} \text {. }
$$

Let $x_{2}(n)=\exp \{y(n)\}$; then

$$
\begin{aligned}
y(n+1) & \leq y(n)+\frac{c(n)}{\beta(n)}-r_{2}(n) \\
& \leq \sum_{s=0}^{n} f(s) y(s)+\frac{c^{M}}{\beta^{L}}-r_{2}^{L},
\end{aligned}
$$

where

$$
f(s)= \begin{cases}0, & 0 \leq s \leq n-1, \\ 1, & s=n\end{cases}
$$

Since

$$
\frac{c^{M}}{\beta^{L}}-r_{2}^{L} \geq \frac{c^{L}}{\beta^{M}}\left(1-\alpha^{M}\right)-r_{2}^{M}>0
$$

by applying Lemma 4, we have

$$
y(n+1) \leq 2\left(\frac{c^{M}}{\beta^{L}}-r_{2}^{L}\right) .
$$

Thus,

$$
\lim _{n \rightarrow+\infty} \sup x_{2}(n) \leq \exp \left\{2\left(\frac{c^{M}}{\beta^{L}}-r_{2}^{L}\right)\right\}:=x_{2}^{*} .
$$


By the second equation of system (6), we can get that

$$
\begin{aligned}
& x_{2}(n+1) \\
& =x_{2}(n) \exp \left[-r_{2}(n)\right. \\
& \left.+\frac{c(n) x_{1}(n)}{\alpha(n)+\beta(n) x_{1}(n)+\gamma(n) x_{2}(n)}\right] \\
& =x_{2}(n) \exp \left\{-r_{2}(n)+c(n)\right. \\
& \times\left[\frac{x_{1}(n)}{\alpha(n)+\beta(n) x_{1}(n)+\gamma(n) x_{2}(n)}\right. \\
& \left.\left.-\frac{1}{\beta(n)}+\frac{1}{\beta(n)}\right]\right\} \\
& =x_{2}(n) \exp \left\{\frac{c(n)}{\beta(n)}-r_{2}(n)-\frac{c(n)}{\beta(n)}\right. \\
& \left.\times \frac{\alpha(n)+\gamma(n) x_{2}(n)}{\alpha(n)+\beta(n) x_{1}(n)+\gamma(n) x_{2}(n)}\right\} \\
& =x_{2}(n) \\
& \times \exp \left\{\frac{c(n)}{\beta(n)}\right. \\
& \times\left(1-\frac{\alpha(n)}{\alpha(n)+\beta(n) x_{1}(n)+\gamma(n) x_{2}(n)}\right) \\
& -r_{2}(n)-\frac{c(n)}{\beta(n)} \\
& \left.\times \frac{\gamma(n)}{\alpha(n)+\beta(n) x_{1}(n)+\gamma(n) x_{2}(n)} x_{2}(n)\right\} \\
& \geq x_{2}(n) \exp \left\{\frac{c^{L}}{\beta^{M}}\left(1-\alpha^{M}\right)-r_{2}^{M}-\frac{c^{M} \gamma^{M}}{\beta^{L}} x_{2}(n)\right\} \text {. }
\end{aligned}
$$

According to $\left(\mathrm{H}_{3}\right)$, applying Lemma 3 to inequality (37), one has

$$
\begin{aligned}
& \lim _{n \rightarrow+\infty} \inf x_{2}(n) \\
& \geq \min \left\{T \exp \left\{\frac{c^{L}\left(1-\alpha^{M}\right)}{\beta^{M}}-r_{2}^{M}-\frac{c^{M} \gamma^{M}}{\beta^{L}} x_{2}^{*}\right\}, T\right\} \\
& :=x_{2 *} .
\end{aligned}
$$

The proof of the theorem is completed.

\section{Existence of Globally Attractive Almost Periodic Solutions}

In this section, we study the existence of a globally attractive almost periodic sequence solution of system (6).
First, we denote by $\Omega$ the set of all solutions $\left(x_{1}(n), x_{2}(n)\right)$ of system (6) satisfying $x_{i *} \leq x_{i}(n) \leq x_{i}^{*}, i=1,2$ for all $n \in Z^{+}$.

In order to apply Lemma 8, we should prove that there exists a bounded solution of system (6) and then construct an adaptive Lyapunov functional for system (6).

Theorem 10. Assume that $\left(H_{1}\right),\left(H_{2}\right)$, and $\left(H_{3}\right)$ hold. Then $\Omega \neq \emptyset$.

Proof. By the almost periodicity of $\left\{r_{1}(n)\right\},\left\{r_{2}(n)\right\},\{K(n)\}$, $\{b(n)\},\{c(n)\},\{\alpha(n)\},\{\beta(n)\}$, and $\{\gamma(n)\}$, there exists an integer valued sequence $\tau_{p}$ with $\tau_{p} \rightarrow \infty$ as $p \rightarrow \infty$ such that

$$
\begin{aligned}
r_{1}\left(n+\tau_{p}\right) \longrightarrow r_{1}(n), & r_{2}\left(n+\tau_{p}\right) \longrightarrow r_{2}(n), \\
K\left(n+\tau_{p}\right) \longrightarrow K(n), & b\left(n+\tau_{p}\right) \longrightarrow b(n), \\
c\left(n+\tau_{p}\right) \longrightarrow c(n), & \alpha\left(n+\tau_{p}\right) \longrightarrow \alpha(n), \\
\beta\left(n+\tau_{p}\right) \longrightarrow \beta(n), & \gamma\left(n+\tau_{p}\right) \longrightarrow \gamma(n)
\end{aligned}
$$

as $p \rightarrow \infty$ uniformly on $Z^{+}$.

Let $\epsilon$ be an arbitrary small positive number. It follows from Theorem 9 that there exists a positive integer $N_{0}$ such that

$$
x_{i *}-\epsilon \leq x_{i}(n) \leq x_{i}^{*}+\epsilon, \quad i=1,2, \forall n>N_{0} .
$$

Write $x_{i p}(n)=x_{i}\left(n+\tau_{p}\right), i=1,2$, for $n \geq N_{0}-\tau_{p}, p=$ $1,2, \ldots$. For any positive integer $q$, it is easy to see that there exist sequence $\left\{x_{i p}: p \geq q, i=1,2\right\}$ such that the sequence $\left\{x_{i p}\right\}$ have subsequences, denoted by $x_{i p}$ again, converging on any finite interval of $Z$ as $p \rightarrow \infty$, respectively. Thus we have sequence $y_{i}(n)$ such that

$$
x_{i p} \longrightarrow y_{i}(n), \quad i=1,2 \text { for } n \in Z^{+} \text {as } p \longrightarrow \infty \text {. }
$$

Combined with

$$
\begin{aligned}
& x_{1 p}(n+1) \\
& =x_{1 p}(n) \exp \left[r_{1}\left(n+\tau_{p}\right)\left(1-\frac{x_{1 p}(n)}{K\left(n+\tau_{p}\right)}\right)\right. \\
& -b\left(n+\tau_{p}\right) x_{2 p}(n) \\
& \times\left(\alpha\left(n+\tau_{p}\right)+\beta\left(n+\tau_{p}\right) x_{1 p}(n)\right. \\
& \left.\left.\quad+\gamma\left(n+\tau_{p}\right) x_{2 p}(n)\right)^{-1}\right],
\end{aligned}
$$




$$
\begin{aligned}
& x_{2 p}(n+1) \\
& =x_{2 p}(n) \exp \left[-r_{2}\left(n+\tau_{p}\right)\right. \\
& +c\left(n+\tau_{p}\right) x_{1 p}(n) \\
& \times\left(\alpha\left(n+\tau_{p}\right)+\beta\left(n+\tau_{p}\right) x_{1 p}(n)\right. \\
& \left.\left.\quad+\gamma\left(n+\tau_{p}\right) x_{2 p}(n)\right)^{-1}\right],
\end{aligned}
$$

it gives

$$
\begin{aligned}
y_{1}(n+1)=y_{1}(n) \exp [ & r_{1}(n)\left(1-\frac{y_{1}(n)}{K(n)}\right) \\
& \left.-\frac{b(n) y_{2}(n)}{\alpha(n)+\beta(n) y_{1}(n)+\gamma(n) y_{2}(n)}\right], \\
y_{2}(n+1)=y_{2}(n) \exp [ & -r_{2}(n) \\
& \left.+\frac{c(n) y_{1}(n)}{\alpha(n)+\beta(n) y_{1}(n)+\gamma(n) y_{2}(n)}\right] .
\end{aligned}
$$

We can easily see that $\left(y_{1}(n), y_{2}(n)\right)$ is a solution of system (6); furthermore,

$$
x_{i *}-\epsilon \leq y_{i}(n) \leq x_{i}^{*}+\epsilon, \quad i=1,2 \text {, for } n \in Z^{+} .
$$

Since $\epsilon$ is an arbitrary small positive number, it follows that

$$
x_{i *} \leq y_{i}(n) \leq x_{i}^{*}, \quad i=1,2 \text {, for } n \in Z^{+} .
$$

The proof of theorem is completed.

In the following, we denote

$$
\begin{gathered}
\forall x \in\left[x_{1 *}, x_{1}^{*}\right], \quad \forall y \in\left[x_{2 *}, x_{2}^{*}\right], \\
g(n, x, y)=\alpha(n)+\beta(n) x+\gamma(n) y, \\
H=\max \{g(n, x, y)\}, \quad h=\min \{g(n, x, y)\}, \\
\eta_{1}=\frac{2 r_{1}^{L} x_{1 *}}{K^{M}+\frac{2 r_{1}^{L} b^{L} \beta^{L} x_{2 *} x_{1 *}^{2}}{K^{M} H^{2}}} \\
-\frac{r_{1}^{M} b^{M} \alpha^{M} x_{1}^{*} x_{2}^{* 2}+r_{1}^{M} b^{M} \beta^{M} x_{1}^{* 2} x_{2}^{* 2}}{K^{L} h^{2}} \\
-\left(\left(\left(b^{M^{2}} \beta^{M^{2}}+c^{M^{2}} \gamma^{M^{2}}\right) x_{1}^{* 2} x_{2}^{* 2}\right.\right. \\
\left.\left.+2 c^{M} \alpha^{M} \gamma^{M} x_{1}^{* 2} x_{2}^{*}+c^{M^{2}} \alpha^{M} x_{1}^{* 2}\right) \times\left(h^{4}\right)^{-1}\right) \\
-\frac{c^{M} \alpha^{M} x_{1}^{*}+\left(c^{M} \gamma^{M}+2 b^{M} \beta^{M}\right) x_{1}^{*} x_{2}^{*}}{h^{2}},
\end{gathered}
$$

$$
\begin{gathered}
\eta_{2}=\frac{2 c^{L} \gamma^{L} x_{1 *} x_{2 *}}{H^{2}}-\frac{r_{1}^{M} b^{M} \alpha^{M} x_{1}^{*} x_{2}^{* 2}+r_{1}^{M} b^{M} \beta^{M} x_{1}^{* 2} x_{2}^{* 2}}{K^{L} h^{2}} \\
-\left(\left(\alpha^{M^{2}} b^{M^{2}} x_{2}^{* 4}+2 b^{M^{2}} \alpha^{M} \beta^{M} x_{1}^{*} x_{2}^{* 4}\right.\right. \\
\left.\left.\quad+b^{M^{2}} \beta^{M^{2}} x_{1}^{* 2} x_{2}^{* 4}+c^{M} \gamma^{M} x_{1}^{* 2} x_{2}^{* 2}\right) \times\left(h^{4}\right)^{-1}\right) \\
-\frac{c^{M} \alpha^{M} x_{1}^{*}+c^{M} \gamma^{M} x_{1}^{*} x_{2}^{*}}{h^{2}}, \\
\eta=\min \left\{\eta_{1}, \eta_{2}\right\} .
\end{gathered}
$$

Theorem 11. Suppose the conditions $\left(H_{1}\right),\left(H_{2}\right)$, and $\left(H_{3}\right)$ are satisfied, and let $0<\eta<1$; then there exists a uniqueness uniformly asymptotically stable almost periodic solution $X=$ $\left(x_{1}(n), x_{2}(n)\right)$ of system (6) which is bounded by $\Omega$ for all $n \in$ $Z^{+}$.

Proof. Let $x_{1}(n)=\exp \left\{p_{1}(n)\right\}, x_{2}(n)=\exp \left\{p_{2}(n)\right\}$. From system (6), we have

$$
\begin{aligned}
& p_{1}(n+1)=p_{1}(n) \\
& \quad+r_{1}(n)\left(1-\frac{e^{p_{1}(n)}}{K(n)}\right)-\frac{b(n) e^{p_{2}(n)}}{g\left(n, e^{p_{1}(n)}, e^{p_{2}(n)}\right)}, \\
& p_{2}(n+1)=p_{2}(n)-r_{2}(n)+\frac{c(n) e^{p_{1}(n)}}{g\left(n, e^{p_{1}(n)}, e^{p_{2}(n)}\right)} .
\end{aligned}
$$

From Theorem 11, we know that the system (47) have bounded solution $Y(n)=\left(p_{1}(n), p_{2}(n)\right)$ satisfying

$$
\ln x_{i *} \leq p_{i}(n) \leq \ln x_{i}^{*}, \quad i=1,2, n \in Z^{+} .
$$

Hence, $\left|p_{i}(n)\right| \leq A_{i}$, where $A_{i}=\max \left\{\left|\ln x_{i *}\right|,\left|\ln x_{i}^{*}\right|\right\}$, $i=1,2$. For $\left(p_{1}(n), p_{2}(n)\right) \in R^{2}$, we define the norm $\left\|\left(p_{1}(n), p_{2}(n)\right)\right\|=\left|p_{1}(n)\right|+\left|p_{2}(n)\right|$.

Consider the product system of system (47)

$$
\begin{aligned}
p_{1}(n+1)= & p_{1}(n)+r_{1}(n)\left(1-\frac{e^{p_{1}(n)}}{K(n)}\right) \\
& -\frac{b(n) e^{p_{2}(n)}}{g\left(n, e^{p_{1}(n)}, e^{p_{2}(n)}\right)}, \\
p_{2}(n+1)= & p_{2}(n)-r_{2}(n)+\frac{c(n) e^{p_{1}(n)}}{g\left(n, e^{p_{1}(n)}, e^{p_{2}(n)}\right)}, \\
q_{1}(n+1)= & q_{1}(n)+r_{1}(n)\left(1-\frac{e^{q_{1}(n)}}{K(n)}\right) \\
& -\frac{b(n) e^{q_{2}(n)}}{g\left(n, e^{q_{1}(n)}, e^{q_{2}(n)}\right)}, \\
q_{2}(n+1)= & q_{2}(n)-r_{2}(n)+\frac{c(n) e^{q_{1}(n)}}{g\left(n, e^{q_{1}(n)}, e^{q_{2}(n)}\right)} .
\end{aligned}
$$


Suppose $X=\left(p_{1}(n), p_{2}(n)\right), Y=\left(q_{1}(n), q_{2}(n)\right)$ are any two solutions of system (49) defined on $Z^{+} \times S^{*} \times S^{*}$; then $\|X\| \leq$ $A,\|Y\| \leq A$, where $A=A_{1}+A_{2}$,

$$
\begin{gathered}
S^{*}=\left\{\left(p_{1}(n), p_{2}(n)\right) \mid \ln x_{i *} \leq p_{i}(n) \leq \ln x_{i}^{*},\right. \\
\left.i=1,2, n \in Z^{+}\right\} .
\end{gathered}
$$
follows:

Consider a Lyapunov function defined on $Z^{+} \times S^{*} \times S^{*}$ as

$$
V(n, X, Y)=\sum_{i=1}^{2}\left(p_{i}(n)-q_{i}(n)\right)^{2}
$$

It is easy to see that the norm $\|X-Y\|=\sum_{i=1}^{2} \mid\left(p_{i}(n)-\right.$ $\left.q_{i}(n)\right) \mid$ and the norm $\|X-Y\|_{*}=\sqrt{\sum_{i=1}^{2}\left(p_{i}(n)-q_{i}(n)\right)^{2}}$ are equivalent; that is, there exist two constants $C_{1}>0, C_{2}>0$, such that

$$
C_{1}\|X-Y\| \leq\|X-Y\|_{*} \leq C_{2}\|X-Y\|
$$

then

$$
\left(C_{1}\|X-Y\|\right)^{2} \leq V(n, X, Y) \leq\left(C_{2}\|X-Y\|\right)^{2} .
$$

Let $a \in C\left(R^{+}, R^{+}\right), a(x)=C_{1}^{2} x^{2}, b \in C\left(R^{+}, R^{+}\right)$, and $b(x)=C_{2}^{2} x^{2}$; thus the condition $\left[C_{1}\right]$ in Lemma 8 is satisfied.

In addition,

$$
\begin{aligned}
& |V(n, X, Y)-V(n, \widetilde{X}, \widetilde{Y})| \\
& =\left|\sum_{i=1}^{2}\left(p_{i}(n)-q_{i}(n)\right)^{2}-\sum_{i=1}^{2}\left(\widetilde{p}_{i}(n)-\widetilde{q}_{i}(n)\right)^{2}\right| \\
& \leq\left|\left(p_{1}(n)-q_{1}(n)\right)^{2}-\left(\tilde{p}_{1}(n)-\tilde{q}_{1}(n)\right)^{2}\right| \\
& +\left|\left(p_{2}(n)-q_{2}(n)\right)^{2}-\left(\tilde{p}_{2}(n)-\widetilde{q}_{2}(n)\right)^{2}\right| \\
& =\left|p_{1}(n)-q_{1}(n)+\tilde{p}_{1}(n)-\tilde{q}_{1}(n)\right| \\
& \times\left|p_{1}(n)-q_{1}(n)-\widetilde{p}_{1}(n)+\widetilde{q}_{1}(n)\right| \\
& +\left|p_{2}(n)-q_{2}(n)+\widetilde{p}_{2}(n)-\widetilde{q}_{2}(n)\right| \\
& \times\left|p_{2}(n)-q_{2}(n)-\widetilde{p}_{2}(n)+\widetilde{q}_{2}(n)\right| \\
& \leq\left(\left|p_{1}(n)\right|+\left|q_{1}(n)\right|+\left|\tilde{p}_{1}(n)\right|+\left|\tilde{q}_{1}(n)\right|\right) \\
& \times\left(\left|p_{1}(n)-\widetilde{p}_{1}(n)\right|+\left|q_{1}(n)-\tilde{q}_{1}(n)\right|\right) \\
& +\left(\left|p_{2}(n)\right|+\left|q_{2}(n)\right|+\left|\widetilde{p}_{2}(n)\right|+\left|\widetilde{q}_{2}(n)\right|\right) \\
& \times\left(\left|p_{2}(n)-\tilde{p}_{2}(n)\right|+\left|q_{2}(n)-\tilde{q}_{2}(n)\right|\right) \\
& \leq L\left\{\left|p_{1}(n)-\tilde{p}_{1}(n)\right|+\left|q_{1}(n)-\tilde{q}_{1}(n)\right|\right. \\
& \left.+\left|p_{2}(n)-\tilde{p}_{2}(n)\right|+\left|q_{2}(n)-\tilde{q}_{2}(n)\right|\right\} \\
& =L\{\|X-\widetilde{X}\|+\|Y-\tilde{Y}\|\} \text {, }
\end{aligned}
$$

where $L=4 \max \left\{A_{1}, A_{2}\right\}$. Hence, the condition $\left[\mathrm{C}_{2}\right]$ of Lemma 8 is satisfied.
Finally, calculate the $\Delta V$ of $V(n)$ along the solutions of (49); we can obtain

$$
\begin{aligned}
\Delta V_{(49)}(n, X, Y)= & V(n+1, X, Y)-V(n, X, Y) \\
= & \sum_{i=1}^{2}\left(p_{i}(n+1)-q_{i}(n+1)\right)^{2} \\
& -\sum_{i=1}^{2}\left(p_{i}(n)-q_{i}(n)\right)^{2} \\
= & \left(p_{1}(n+1)-q_{1}(n+1)\right)^{2} \\
& -\left(p_{1}(n)-q_{1}(n)\right)^{2} \\
& +\left(p_{2}(n+1)-q_{2}(n+1)\right)^{2} \\
& -\left(p_{2}(n)-q_{2}(n)\right)^{2} .
\end{aligned}
$$

In the view of (49), we get

$$
\begin{aligned}
& \left(p_{1}(n+1)-q_{1}(n+1)\right)^{2}-\left(p_{1}(n)-q_{1}(n)\right)^{2} \\
& =\left[\left(p_{1}(n)-q_{1}(n)\right)-\frac{r_{1}(n)}{K(n)}\left(e^{p_{1}(n)}-e^{q_{1}(n)}\right)\right. \\
& \left.-b(n)\left(\frac{e^{p_{2}(n)}}{g\left(n, e^{p_{1}(n)}, e^{p_{2}(n)}\right)}-\frac{e^{q_{2}(n)}}{g\left(n, e^{q_{1}(n)}, e^{q_{2}(n)}\right)}\right)\right]^{2} \\
& -\left(p_{1}(n)-q_{1}(n)\right)^{2} \\
& =\left[\left(p_{1}(n)-q_{1}(n)\right)-\frac{r_{1}(n)}{K(n)}\left(e^{p_{1}(n)}-e^{q_{1}(n)}\right)\right. \\
& -b(n)\left(\frac{e^{p_{2}(n)}}{g\left(n, e^{p_{1}(n)}, e^{p_{2}(n)}\right)}-\frac{e^{p_{2}(n)}}{g\left(n, e^{q_{1}(n)}, e^{q_{2}(n)}\right)}\right. \\
& \left.\left.+\frac{e^{p_{2}(n)}}{g\left(n, e^{q_{1}(n)}, e^{q_{2}(n)}\right)}-\frac{e^{q_{2}(n)}}{g\left(n, e^{q_{1}(n)}, e^{q_{2}(n)}\right)}\right)\right]^{2} \\
& -\left(p_{1}(n)-q_{1}(n)\right)^{2} \\
& =\left[\left(p_{1}(n)-q_{1}(n)\right)\right. \\
& -\frac{b(n) e^{p_{2}(n)}\left(\alpha(n)+\beta(n) e^{p_{1}(n)}\right)}{g\left(n, e^{q_{1}(n)}, e^{q_{2}(n)}\right) g\left(n, e^{p_{1}(n)}, e^{p_{2}(n)}\right)} \\
& \times\left(e^{p_{2}(n)}-e^{q_{2}(n)}\right) \\
& -\left(\frac{r_{1}(n)}{K(n)}-\frac{b(n) e^{p_{2}(n)} \beta(n)}{g\left(n, e^{q_{1}(n)}, e^{q_{2}(n)}\right) g\left(n, e^{p_{1}(n)}, e^{p_{2}(n)}\right)}\right) \\
& \left.\times\left(e^{p_{1}(n)}-e^{q_{1}(n)}\right)\right]^{2}-\left(p_{1}(n)-q_{1}(n)\right)^{2}
\end{aligned}
$$


8

Abstract and Applied Analysis

$$
\begin{aligned}
& =\frac{b^{2}(n) e^{2 p_{2}(n)}\left(\alpha(n)+\beta(n) e^{p_{1}(n)}\right)^{2}}{g^{2}\left(n, e^{q_{1}(n)}, e^{q_{2}(n)}\right) g^{2}\left(n, e^{p_{1}(n)}, e^{p_{2}(n)}\right)} \\
& \times\left(e^{p_{2}(n)}-e^{q_{2}(n)}\right)^{2} \\
& +\left(\frac{r_{1}(n)}{K(n)}-\frac{b(n) e^{p_{2}(n)} \beta(n)}{g\left(n, e^{q_{1}(n)}, e^{q_{2}(n)}\right) g\left(n, e^{p_{1}(n)}, e^{p_{2}(n)}\right)}\right)^{2} \\
& \times\left(e^{p_{1}(n)}-e^{q_{1}(n)}\right)^{2} \\
& -2 \frac{b(n) e^{p_{2}(n)}\left(\alpha(n)+\beta(n) e^{p_{1}(n)}\right)}{g\left(n, e^{q_{1}(n)}, e^{q_{2}(n)}\right) g\left(n, e^{p_{1}(n)}, e^{p_{2}(n)}\right)} \\
& \times\left(e^{p_{2}(n)}-e^{q_{2}(n)}\right)\left(p_{1}(n)-q_{1}(n)\right) \\
& -2\left(\frac{r_{1}(n)}{K(n)}-\frac{b(n) e^{p_{2}(n)} \beta(n)}{g\left(n, e^{q_{1}(n)}, e^{q_{2}(n)}\right) g\left(n, e^{p_{1}(n)}, e^{p_{2}(n)}\right)}\right) \\
& \times\left(e^{p_{1}(n)}-e^{q_{1}(n)}\right)\left(p_{1}(n)-q_{1}(n)\right) \\
& +2\left(\frac{r_{1}(n)}{K(n)}-\frac{b(n) e^{p_{2}(n)} \beta(n)}{g\left(n, e^{q_{1}(n)}, e^{q_{2}(n)}\right) g\left(n, e^{p_{1}(n)}, e^{p_{2}(n)}\right)}\right) \\
& \times \frac{b(n) e^{p_{2}(n)}\left(\alpha(n)+\beta(n) e^{p_{1}(n)}\right)}{g\left(n, e^{q_{1}(n)}, e^{q_{2}(n)}\right) g\left(n, e^{p_{1}(n)}, e^{p_{2}(n)}\right)} \\
& \times\left(e^{p_{1}(n)}-e^{q_{1}(n)}\right)\left(e^{p_{2}(n)}-e^{q_{2}(n)}\right), \\
& \left(p_{2}(n+1)-q_{2}(n+1)\right)^{2}-\left(p_{2}(n)-q_{2}(n)\right)^{2}
\end{aligned}
$$$$
=\left[\left(p_{2}(n)-q_{2}(n)\right)+c(n)\right.
$$$$
\left.\times\left(\frac{e^{p_{1}(n)}}{g\left(n, e^{p_{1}(n)}, e^{p_{2}(n)}\right)}-\frac{e^{q_{1}(n)}}{g\left(n, e^{q_{1}(n)}, e^{q_{2}(n)}\right)}\right)\right]^{2}
$$$$
-\left(p_{2}(n)-q_{2}(n)\right)^{2}
$$$$
=\left[\left(p_{2}(n)-q_{2}(n)\right)\right.
$$$$
+c(n)\left(\frac{e^{p_{1}(n)}}{g\left(n, e^{p_{1}(n)}, e^{p_{2}(n)}\right)}-\frac{e^{p_{1}(n)}}{g\left(n, e^{q_{1}(n)}, e^{q_{2}(n)}\right)}\right.
$$$$
\left.\left.+\frac{e^{p_{1}(n)}}{g\left(n, e^{q_{1}(n)}, e^{q_{2}(n)}\right)}-\frac{e^{q_{1}(n)}}{g\left(n, e^{q_{1}(n)}, e^{q_{2}(n)}\right)}\right)\right]^{2}
$$$$
-\left(p_{2}(n)-q_{2}(n)\right)^{2}
$$$$
=\left[\left(p_{2}(n)-q_{2}(n)\right)\right.
$$$$
+\frac{c(n) \alpha(n)+c(n) \gamma(n) e^{p_{2}(n)}}{g\left(n, e^{q_{1}(n)}, e^{q_{2}(n)}\right) g\left(n, e^{p_{1}(n)}, e^{p_{2}(n)}\right)}
$$$$
\times\left(e^{p_{1}(n)}-e^{q_{1}(n)}\right)
$$

Substituting (56) into (55), we have

$$
\begin{aligned}
& \Delta V_{(49)}(n, X, Y) \\
&=V(n+1, X, Y)-V(n, X, Y) \\
&=\left[\left(\frac{r_{1}(n)}{K(n)}-\frac{b(n) e^{p_{2}(n)} \beta(n)}{g\left(n, e^{q_{1}(n)}, e^{q_{2}(n)}\right) g\left(n, e^{p_{1}(n)}, e^{p_{2}(n)}\right)}\right)^{2}\right. \\
&\left.+\frac{\left(c(n) \alpha(n)+c(n) \gamma(n) e^{p_{2}(n)}\right)^{2}}{g^{2}\left(n, e^{q_{1}(n)}, e^{q_{2}(n)}\right) g^{2}\left(n, e^{p_{1}(n)}, e^{p_{2}(n)}\right)}\right] \\
& \times\left(e^{p_{1}(n)}-e^{q_{1}(n)}\right)^{2} \\
&+\left(\left(b^{2}(n) e^{2 p_{2}(n)}\left(\alpha(n)+\beta(n) e^{p_{1}(n)}\right)^{2}\right.\right. \\
&\left.\quad+c^{2}(n) \gamma^{2}(n) e^{2 p_{1}(n)}\right) \\
&\left.\quad \times\left(g^{2}\left(n, e^{q_{1}(n)}, e^{q_{2}(n)}\right) g^{2}\left(n, e^{p_{1}(n)}, e^{p_{2}(n)}\right)\right)^{-1}\right) \\
& \times\left(e^{p_{2}(n)}-e^{q_{2}(n)}\right)^{2}
\end{aligned}
$$


Abstract and Applied Analysis

9

$$
\begin{aligned}
& +2\left[\left(\frac{r_{1}(n)}{K(n)}-\frac{b(n) e^{p_{2}(n)} \beta(n)}{g\left(n, e^{q_{1}(n)}, e^{q_{2}(n)}\right) g\left(n, e^{p_{1}(n)}, e^{p_{2}(n)}\right)}\right)\right. \\
& \times \frac{b(n) e^{p_{2}(n)}\left(\alpha(n)+\beta(n) e^{p_{1}(n)}\right)}{g\left(n, e^{q_{1}(n)}, e^{q_{2}(n)}\right) g\left(n, e^{p_{1}(n)}, e^{p_{2}(n)}\right)} \\
& \left.-\frac{\left(c(n) \alpha(n)+c(n) \gamma(n) e^{p_{2}(n)}\right)\left(c(n) \gamma(n) e^{p_{1}(n)}\right)}{g^{2}\left(n, e^{q_{1}(n)}, e^{q_{2}(n)}\right) g^{2}\left(n, e^{p_{1}(n)}, e^{p_{2}(n)}\right)}\right] \\
& \times\left(e^{p_{1}(n)}-e^{q_{1}(n)}\right)\left(e^{p_{2}(n)}-e^{q_{2}(n)}\right) \\
& -2 \frac{b(n) e^{p_{2}(n)}\left(\alpha(n)+\beta(n) e^{p_{1}(n)}\right)}{g\left(n, e^{q_{1}(n)}, e^{q_{2}(n)}\right) g\left(n, e^{p_{1}(n)}, e^{p_{2}(n)}\right)} \\
& \times\left(e^{p_{2}(n)}-e^{q_{2}(n)}\right)\left(p_{1}(n)-q_{1}(n)\right) \\
& -2\left(\frac{r_{1}(n)}{K(n)}-\frac{b(n) e^{p_{2}(n)} \beta(n)}{g\left(n, e^{q_{1}(n)}, e^{q_{2}(n)}\right) g\left(n, e^{p_{1}(n)}, e^{p_{2}(n)}\right)}\right) \\
& \times\left(e^{p_{1}(n)}-e^{q_{1}(n)}\right)\left(p_{1}(n)-q_{1}(n)\right) \\
& -2 \frac{c(n) \gamma(n) e^{p_{1}(n)}}{g\left(n, e^{q_{1}(n)}, e^{q_{2}(n)}\right) g\left(n, e^{p_{1}(n)}, e^{p_{2}(n)}\right)} \\
& \times\left(e^{p_{2}(n)}-e^{q_{2}(n)}\right)\left(p_{2}(n)-q_{2}(n)\right) \\
& +2 \frac{c(n) \alpha(n)+c(n) \gamma(n) e^{p_{2}(n)}}{g\left(n, e^{q_{1}(n)}, e^{q_{2}(n)}\right) g\left(n, e^{p_{1}(n)}, e^{p_{2}(n)}\right)} \\
& \times\left(e^{p_{1}(n)}-e^{q_{1}(n)}\right)\left(p_{2}(n)-q_{2}(n)\right) .
\end{aligned}
$$

Using the mean value theorem, we get

$$
e^{p_{i}(n)}-e^{q_{i}(n)}=\xi_{i}(n)\left(p_{i}(n)-q_{i}(n)\right), \quad i=1,2,
$$

where $\xi_{i}(n)$ lies between $e^{p_{i}(n)}$ and $e^{q_{i}(n)}, i=1,2$. From (57) and (58), we have

$$
\begin{aligned}
\Delta V_{(49)} & (n, X, Y) \\
= & V(n+1, X, Y)-V(n, X, Y) \\
= & {\left[\left(\frac{r_{1}(n)}{K(n)}-\frac{b(n) e^{p_{2}(n)} \beta(n)}{g\left(n, e^{q_{1}(n)}, e^{q_{2}(n)}\right) g\left(n, e^{p_{1}(n)}, e^{p_{2}(n)}\right)}\right)^{2} \xi_{1}^{2}(n)\right.} \\
& +\frac{\left(c(n) \alpha(n)+c(n) \gamma(n) e^{p_{2}(n)}\right)^{2}}{g^{2}\left(n, e^{q_{1}(n)}, e^{q_{2}(n)}\right) g^{2}\left(n, e^{p_{1}(n)}, e^{p_{2}(n)}\right)} \xi_{1}^{2}(n)-2 \\
& \times\left(\frac{r_{1}(n)}{K(n)}-\frac{b(n) e^{p_{2}(n)} \beta(n)}{g\left(n, e^{q_{1}(n)}, e^{q_{2}(n)}\right) g\left(n, e^{p_{1}(n)}, e^{p_{2}(n)}\right)}\right) \\
& \left.\times \xi_{1}(n)\right]\left(p_{1}(n)-q_{1}(n)\right)^{2}
\end{aligned}
$$

$$
\begin{aligned}
& +\left[\left(\left(b^{2}(n) e^{2 p_{2}(n)}\left(\alpha(n)+\beta(n) e^{p_{1}(n)}\right)^{2}\right.\right.\right. \\
& \left.+c^{2}(n) \gamma^{2}(n) e^{2 p_{1}(n)}\right) \\
& \left.\times\left(g^{2}\left(n, e^{q_{1}(n)}, e^{q_{2}(n)}\right) g^{2}\left(n, e^{p_{1}(n)}, e^{p_{2}(n)}\right)\right)^{-1}\right) \xi_{2}^{2}(n) \\
& \left.-2 \frac{c(n) \gamma(n) e^{p_{1}(n)}}{g\left(n, e^{q_{1}(n)}, e^{q_{2}(n)}\right) g\left(n, e^{p_{1}(n)}, e^{p_{2}(n)}\right)} \xi_{2}\right] \\
& \times\left(p_{2}(n)-q_{2}(n)\right)^{2} \\
& +2\left[\left(\frac{r_{1}(n)}{K(n)}-\frac{b(n) e^{p_{2}(n)} \beta(n)}{g\left(n, e^{q_{1}(n)}, e^{q_{2}(n)}\right) g\left(n, e^{p_{1}(n)}, e^{p_{2}(n)}\right)}\right)\right. \\
& \times \frac{b(n) e^{p_{2}(n)}\left(\alpha(n)+\beta(n) e^{p_{1}(n)}\right)}{g\left(n, e^{q_{1}(n)}, e^{q_{2}(n)}\right) g\left(n, e^{p_{1}(n)}, e^{p_{2}(n)}\right)} \xi_{1}(n) \xi_{2}(n) \\
& -\frac{\left(c(n) \alpha(n)+c(n) \gamma(n) e^{p_{2}(n)}\right)\left(c(n) \gamma(n) e^{p_{1}(n)}\right)}{g^{2}\left(n, e^{q_{1}(n)}, e^{q_{2}(n)}\right) g^{2}\left(n, e^{p_{1}(n)}, e^{p_{2}(n)}\right)} \\
& \times \xi_{1}(n) \xi_{2}(n) \\
& -\frac{b(n) e^{p_{2}(n)}\left(\alpha(n)+\beta(n) e^{p_{1}(n)}\right)}{g\left(n, e^{q_{1}(n)}, e^{q_{2}(n)}\right) g\left(n, e^{p_{1}(n)}, e^{p_{2}(n)}\right)} \xi_{2}(n) \\
& \left.+\frac{c(n) \alpha(n)+c(n) \gamma(n) e^{p_{2}(n)}}{g\left(n, e^{q_{1}(n)}, e^{q_{2}(n)}\right) g\left(n, e^{p_{1}(n)}, e^{p_{2}(n)}\right)} \xi_{1}(n)\right] \\
& \times\left(p_{1}(n)-q_{1}(n)\right)\left(p_{2}(n)-q_{2}(n)\right),
\end{aligned}
$$

and we get

$$
\Delta V_{(49)}=V_{1}+V_{2}+V_{3}
$$

where

$$
\begin{aligned}
& V_{1}(n) \\
& =\left[\left(\frac{r_{1}(n)}{K(n)}-\frac{b(n) e^{p_{2}(n)} \beta(n)}{g\left(n, e^{q_{1}(n)}, e^{q_{2}(n)}\right) g\left(n, e^{p_{1}(n)}, e^{p_{2}(n)}\right)}\right)^{2} \xi_{1}^{2}(n)\right. \\
& \quad+\frac{\left(c(n) \alpha(n)+c(n) \gamma(n) e^{p_{2}(n)}\right)^{2}}{g^{2}\left(n, e^{q_{1}(n)}, e^{q_{2}(n)}\right) g^{2}\left(n, e^{p_{1}(n)}, e^{p_{2}(n)}\right)} \xi_{1}^{2}(n) \\
& \quad-2\left(\frac{r_{1}(n)}{K(n)}-\frac{b(n) e^{p_{2}(n)} \beta(n)}{g\left(n, e^{q_{1}(n)}, e^{q_{2}(n)}\right) g\left(n, e^{p_{1}(n)}, e^{p_{2}(n)}\right)}\right) \\
& \left.\quad \times \xi_{1}(n)\right]\left(p_{1}(n)-q_{1}(n)\right)^{2}
\end{aligned}
$$


10

Abstract and Applied Analysis

$$
\begin{aligned}
\leq\left[\left(\left(b^{M^{2}} \beta^{M^{2}}+c^{M^{2}} \gamma^{M^{2}}\right) x_{1}^{* 2} x_{2}^{* 2}+c^{M^{2}} \alpha^{M} x_{1}^{* 2}\right.\right. & \leq 2\left[\frac{c^{M} \alpha^{M} x_{1}^{*}+c^{M} \gamma^{M} x_{1}^{*} x_{2}^{*}}{h^{2}}\right. \\
\left.\left.+2 c^{M} \alpha^{M} \gamma^{M} x_{2}^{*} x_{1}^{* 2}\right) \times\left(h^{4}\right)^{-1}\right)+\frac{2 b^{M} \beta^{M} x_{2}^{*} x_{1}^{*}}{h^{2}} & \left.+\frac{r_{1}^{M} b^{M} \alpha^{M} x_{1}^{*} x_{2}^{* 2}+r_{1}^{M} b^{M} \beta^{M} x_{1}^{* 2} x_{2}^{* 2}}{K^{L} h^{2}}\right] \\
-\frac{2 r_{1}^{L} b^{L} \beta^{L} x_{2 *} x_{1 *}^{2}}{K^{M} H^{2}}+\frac{r_{1}^{M^{2}} x_{1}^{* 2}}{\left.K^{L^{2}}-\frac{2 r_{1}^{L} x_{1 *}}{K^{M}}\right]} & \times\left[\left(p_{1}(n)-q_{1}(n)\right)^{2}+\left(p_{2}(n)-q_{2}(n)\right)^{2}\right] .
\end{aligned}
$$

Hence,

$$
\begin{aligned}
& \Delta V_{(49)} \\
& \leq\left[\left(\left(b^{M^{2}} \beta^{M^{2}}+c^{M^{2}} \gamma^{M^{2}}\right) x_{1}^{* 2} x_{2}^{* 2}+c^{M^{2}} \alpha^{M} x_{1}^{* 2}\right.\right. \\
& \left.\left.+2 c^{M} \alpha^{M} \gamma^{M} x_{2}^{*} x_{1}^{* 2}\right) \times\left(h^{4}\right)^{-1}\right) \\
& +\frac{2 b^{M} \beta^{M} x_{2}^{*} x_{1}^{*}}{h^{2}}-\frac{2 r_{1}^{M} b^{M} \beta^{M} x_{2}^{*} x_{1}^{* 2}}{K^{L} h^{2}}+\frac{r_{1}^{M^{2}} x_{1}^{* 2}}{K^{L^{2}}} \\
& \left.-\frac{2 r_{1}^{L} x_{1 *}}{K^{M}}\right]\left(p_{1}(n)-q_{1}(n)\right)^{2} \\
& +\left[\left(\left(\alpha^{M^{2}} b^{M^{2}} x_{2}^{* 3}+2 b^{M^{2}} \alpha^{M} \beta^{M} x_{1}^{*} x_{2}^{* 3}\right.\right.\right. \\
& \left.\left.+b^{M^{2}} \beta^{M^{2}} x_{1}^{* 2} x_{2}^{* 4}+c^{M} \gamma^{M} x_{1}^{*} x_{2}^{* 2}\right) \times\left(h^{4}\right)^{-1}\right) \\
& \left.-\frac{2 c^{L} \gamma^{L} x_{1 *} x_{2 *}}{H^{2}}\right] \\
& \times\left(p_{2}(n)-q_{2}(n)\right)^{2} \\
& +\left[\frac{c^{M} \alpha^{M} x_{1}^{*}+c^{M} \gamma^{M} x_{1}^{*} x_{2}^{*}}{h^{2}}\right. \\
& \left.+\frac{r_{1}^{M} b^{M} \alpha^{M} x_{1}^{*} x_{2}^{* 2}+r_{1}^{M} b^{M} \beta^{M} x_{1}^{* 2} x_{2}^{* 2}}{K^{L} h^{2}}\right] \\
& \times\left[\left(p_{1}(n)-q_{1}(n)\right)^{2}+\left(p_{2}(n)-q_{2}(n)\right)^{2}\right] \\
& \leq-\left[\frac{2 r_{1}^{L} x_{1 *}}{K^{M}}+\frac{2 r_{1}^{L} b^{L} \beta^{L} x_{2 *} x_{1 *}^{2}}{K^{M} H^{2}}\right. \\
& -\frac{r_{1}^{M} b^{M} \alpha^{M} x_{1}^{*} x_{2}^{* 2}+r_{1}^{M} b^{M} \beta^{M} x_{1}^{* 2} x_{2}^{* 2}}{K^{L} h^{2}} \\
& -\left(\left(\left(b^{M^{2}} \beta^{M^{2}}+c^{M^{2}} \gamma^{M^{2}}\right) x_{1}^{* 2} x_{2}^{* 2}\right.\right. \\
& \left.\left.+2 c^{M} \alpha^{M} \gamma^{M} x_{1}^{* 2} x_{2}^{*}+c^{M^{2}} \alpha^{M} x_{1}^{* 2}\right) \times\left(h^{4}\right)^{-1}\right) \\
& \left.-\frac{c^{M} \alpha^{M} x_{1}^{*}+\left(c^{M} \gamma^{M}+2 b^{M} \beta^{M}\right) x_{1}^{*} x_{2}^{*}}{h^{2}}\right] \\
& \times\left(p_{1}(n)-q_{1}(n)\right)^{2}
\end{aligned}
$$$$
\times\left(p_{1}(n)-q_{1}(n)\right)\left(p_{2}(n)-q_{2}(n)\right)
$$ 


$$
\begin{aligned}
- & {\left[\frac{2 c^{L} \gamma^{L} x_{1 *} x_{2 *}}{H^{2}}-\frac{r_{1}^{M} b^{M} \alpha^{M} x_{1}^{*} x_{2}^{* 2}+r_{1}^{M} b^{M} \beta^{M} x_{1}^{* 2} x_{2}^{* 2}}{K^{L} h^{2}}\right.} \\
& -\left(\left(\alpha^{M^{2}} b^{M^{2}} x_{2}^{* 4}+2 b^{M^{2}} \alpha^{M} \beta^{M} x_{1}^{*} x_{2}^{* 4}\right.\right. \\
& \left.\left.+b^{M^{2}} \beta^{M^{2}} x_{1}^{* 2} x_{2}^{* 4}+c^{M} \gamma^{M} x_{1}^{* 2} x_{2}^{* 2}\right) \times\left(h^{4}\right)^{-1}\right) \\
& \left.-\frac{c^{M} \alpha^{M} x_{1}^{*}+c^{M} \gamma^{M} x_{1}^{*} x_{2}^{*}}{h^{2}}\right]\left(p_{2}(n)-q_{2}(n)\right)^{2} \\
\leq & -\eta_{1}\left(p_{1}(n)-q_{1}(n)\right)^{2}-\eta_{2}\left(p_{2}(n)-q_{2}(n)\right)^{2} \\
\leq & -\eta\left[\left(p_{1}(n)-q_{1}(n)\right)^{2}+\left(p_{2}(n)-q_{2}(n)\right)^{2}\right] \\
= & -\eta V(n, X, Y),
\end{aligned}
$$

where $\eta=\min \left\{\eta_{1}, \eta_{2}\right\}$. That is, there exists a positive constant $0<\eta<1$ such that

$$
\Delta V_{(49)} \leq-\eta V(n, X, Y) .
$$

From $0<\eta<1$, the condition $\left[\mathrm{C}_{3}\right]$ of Lemma 8 is satisfied. So, from Lemma 8, there exists a uniqueness uniformly asymptotically stable almost periodic solution $X(n)=\left(p_{1}(n), p_{2}(n)\right)$ of (47) which is bounded by $S^{*}$ for all $n \in Z^{+}$. Which means that there exists a uniqueness uniformly asymptotically stable almost periodic solution $X(n)=\left(x_{1}(n), x_{2}(n)\right)$ of $(6)$ which is bounded by $\Omega$ for all $n \in Z^{+}$. The proof is completed.

\section{Application}

Example 12. Consider the following discrete model:

$$
\begin{aligned}
& x_{1}(n+1) \\
& =x_{1}(n) \\
& \times \exp \left[(1.1+0.4 \sin (n))\left(1-x_{1}(n)\right)\right. \\
& -(0.3-0.2 \cos (n)) x_{2}(n) \\
& \times(0.3-0.1 \sin (n) \\
& \left.\left.+(0.6+0.1 \cos (n)) x_{1}(n)+x_{2}(n)\right)^{-1}\right] \text {, } \\
& x_{2}(n+1) \\
& =x_{2}(n) \exp [-(0.8+0.2 \sin (n)) \\
& +(1.5-0.3 \sin (n)) x_{1}(n) \\
& \times(0.3-0.1 \sin (n) \\
& \left.\left.+(0.6+0.1 \cos (n)) x_{1}(n)+x_{2}(n)\right)^{-1}\right] \text {, }
\end{aligned}
$$

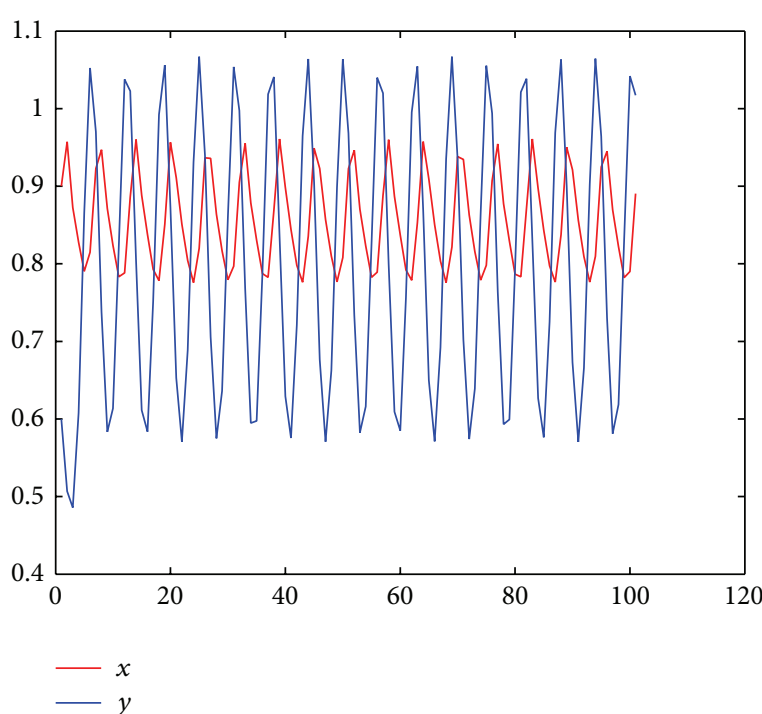

FIGURE 1: The orbit of predator $\left(x_{2}\right)$-prey $\left(x_{1}\right)$.

with initial conditions $(0.9,0.6)$; then system $(64)$ is persistent and has a globally attractive almost periodic solution.

By simple computation, we derive

$$
r_{1}^{L}-\frac{b^{M}}{\gamma^{L}}=0.2>0, \quad \frac{c^{L}}{\beta^{M}}\left(1-\alpha^{M}\right)-r_{2}^{M} \approx 0.2857>0 .
$$

Thus, the system (64) is persistent. Its integral curves and orbits are shown in Figure 1, respectively.

\section{Conflict of Interests}

The authors declare that there is no conflict of interests regarding the publication of this paper.

\section{Acknowledgments}

The authors express their sincere thanks to the anonymous reviewers for their valuable comments and suggestions for improving the quality of the paper. This work is supported by the Natural Science Foundation of China (Grant nos. 11071205 and 11101349), PAPD of Jiangsu Higher Education Institutions, and Postgraduate training project of Jiangsu Normal University and Jiangsu Province.

\section{References}

[1] J. O. Alzabut, J. J. Nieto, and G. T. Stamov, "Existence and exponential stability of positive almost periodic solutions for a model of hematopoiesis," Boundary Value Problems, vol. 2009, Article ID 127510, 10 pages, 2009.

[2] F. Chen, "Almost periodic solution of the non-autonomous two-species competitive model with stage structure," Applied Mathematics and Computation, vol. 181, no. 1, pp. 685-693, 2006.

[3] Z. Du and Y. Lv, "Permanence and almost periodic solution of a Lotka-Volterra model with mutual interference and time 
delays," Applied Mathematical Modelling, vol. 37, no. 3, pp. 10541068, 2013.

[4] C. H. Feng, Y. J. Liu, and W. G. Ge, "Almost periodic solutions for delay Lotka-Volterra competitive systems," Acta Mathematicae Applicatae Sinica, vol. 28, no. 3, pp. 458-465, 2005.

[5] X. Lin and F. D. Chen, "Almost periodic solution for a Volterra model with mutual interference and Beddington-DeAngelis functional response," Applied Mathematics and Computation, vol. 214, no. 2, pp. 548-556, 2009.

[6] C. Shen, "Permanence and global attractivity of the foodchain system with Holling IV type functional response," Applied Mathematics and Computation, vol. 194, no. 1, pp. 179-185, 2007.

[7] W. Qi and B. Dai, "Almost periodic solution for $n$-species LotkaVolterra competitive system with delay and feedback controls," Applied Mathematics and Computation, vol. 200, no. 1, pp. 133146, 2008.

[8] Y. Zhu and K. Wang, "Existence and global attractivity of positive periodic solutions for a predator-prey model with modified Leslie-Gower Holling-type II schemes," Journal of Mathematical Analysis and Applications, vol. 384, no. 2, pp. 400408, 2011.

[9] Z. Cai, L. Huang, and H. Chen, "Positive periodic solution for a multispecies competition-predator system with Holling III functional response and time delays," Applied Mathematics and Computation, vol. 217, no. 10, pp. 4866-4878, 2011.

[10] J. R. Beddington, "Mutual interference between parasites or predators and its effect on searching efficiency," Journal of Animal Ecology, vol. 44, pp. 331-340, 1975.

[11] D. L. DeAngelis, R. A. Goldstein, and R. V. O’Neill, “A model for trophic interaction,” Ecology, vol. 56, pp. 881-892, 1975.

[12] M. Fan and Y. Kuang, "Dynamics of a nonautonomous predator-prey system with the Beddington-DeAngelis functional response," Journal of Mathematical Analysis and Applications, vol. 295, no. 1, pp. 15-39, 2004.

[13] S. Liu, E. Beretta, and D. Breda, "Predator-prey model of Beddington-DeAngelis type with maturation and gestation delays," Nonlinear Analysis: Real World Applications, vol. 11, no. 5, pp. 4072-4091, 2010.

[14] H. K. Baek, "Qualitative analysis of Beddington-DeAngelis type impulsive predator-prey models," Nonlinear Analysis: Real World Applications, vol. 11, no. 3, pp. 1312-1322, 2010.

[15] L. Li, G. Zhang, G. Sun, and Z. Wang, "Existence of periodic positive solutions for a competitive system with two parameters," Journal of Difference Equations and Applications, vol. 20, no. 3, pp. 341-353, 2014.

[16] J. Zhang and J. Wang, "Periodic solutions for discrete predator-prey systems with the Beddington-DeAngelis functional response," Applied Mathematics Letters, vol. 19, no. 12, pp. 1361-1366, 2006.

[17] L. Li and Z. Wang, "Global stability of periodic solutions for a discrete predator-prey system with functional response," Nonlinear Dynamics, vol. 72, no. 3, pp. 507-516, 2013.

[18] Z. Li and F. Chen, "Almost periodic solutions of a discrete almost periodic logistic equation," Mathematical and Computer Modelling, vol. 50, no. 1-2, pp. 254-259, 2009.

[19] C. Y. Niu and X. X. Chen, "Almost periodic sequence solutions of a discrete Lotka-Volterra competitive system with feedback control," Nonlinear Analysis: Real World Applications, vol. 10, no. 5, pp. 3152-3161, 2009.
[20] Y. Li, T. Zhang, and Y. Ye, "On the existence and stability of a unique almost periodic sequence solution in discrete predatorprey models with time delays," Applied Mathematical Modelling, vol. 35, no. 11, pp. 5448-5459, 2011.

[21] F. Chen, "Permanence for the discrete mutualism model with time delays," Mathematical and Computer Modelling, vol. 47, no. 3-4, pp. 431-435, 2008.

[22] W. Yang and X. Li, "Permanence for a delayed discrete ratiodependent predator-prey model with monotonic functional responses," Nonlinear Analysis: Real World Applications, vol. 10, no. 2, pp. 1068-1072, 2009.

[23] D. Cheban and C. Mammana, "Invariant manifolds, global attractors and almost periodic solutions of nonautonomous difference equations," Nonlinear Analysis: Theory, Methods \& Applications, vol. 56, no. 4, pp. 465-484, 2004.

[24] S. N. Zhang, "Existence of almost periodic solution for difference systems," Annals of Differential Equations, vol. 16, pp. 184206, 2000. 


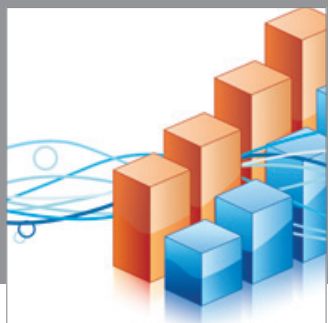

Advances in

Operations Research

mansans

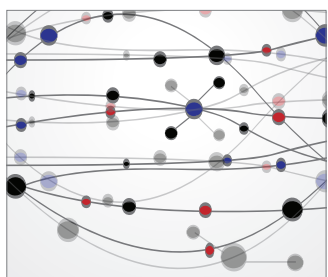

The Scientific World Journal
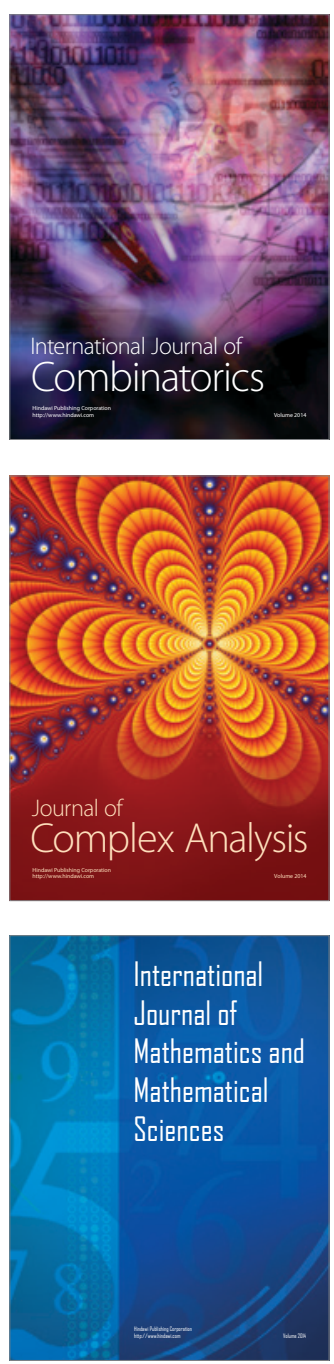
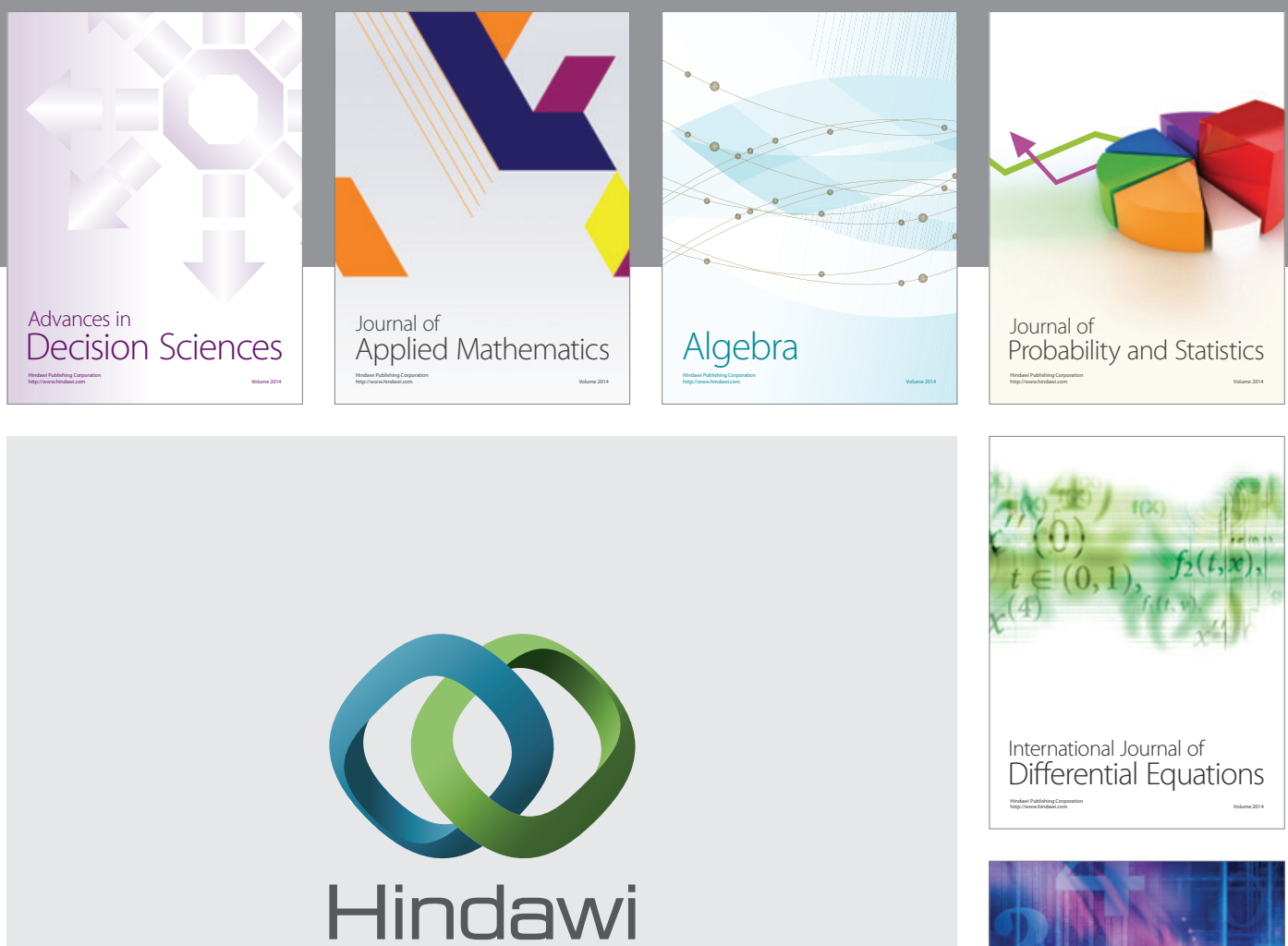

Submit your manuscripts at http://www.hindawi.com
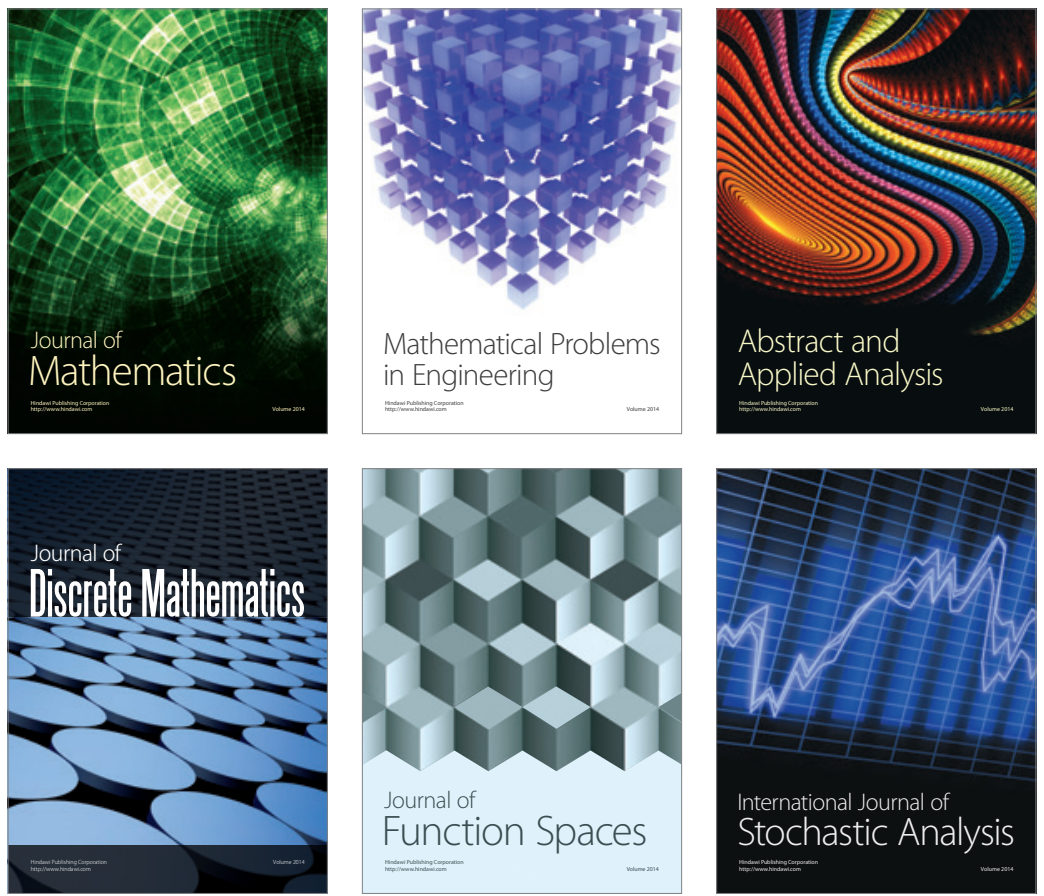

Journal of

Function Spaces

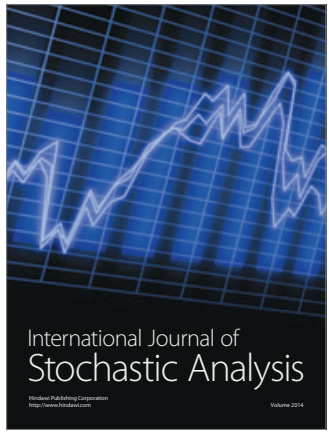

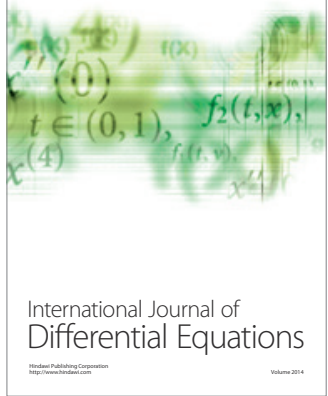
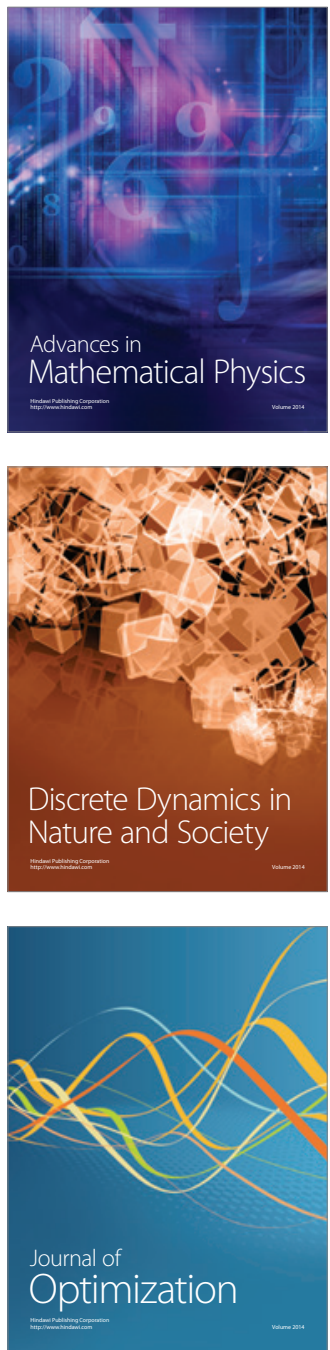